International Journal of Pure and Applied Mathematics

Volume 106 No. 2 2016, 443-452

ISSN: 1311-8080 (printed version); ISSN: 1314-3395 (on-line version)

url: http://www.ijpam.eu

doi: 10.12732/ijpam.v106i2.8

\title{
GENERAL MANPOWER-SCBZ MACHINE SYSTEM WITH MARKOVIAN PRODUCTION GENERAL SALES AND GENERAL RECRUITMENT
}

\author{
M. Sneha Latha ${ }^{1 \S}$, P. Sekar ${ }^{2}$, R. Ramanarayanan ${ }^{3}$ \\ ${ }^{1}$ SRM University \\ Chennai, Tamil Nadu, INDIA \\ ${ }^{2}$ C. Kandaswami Naidu College for Men \\ Anna Nagar, Chennai, Tamil Nadu, INDIA \\ ${ }^{3}$ Vel Tech University \\ Chennai, INDIA
}

\begin{abstract}
Two Manpower planning models with different recruitment patterns are studied. During the operation time a machine produces random number of products. After the operation time, the sale time starts, which has one among two distinct distributions depending on the magnitude of production is within or exceeding a random threshold. In this paper, the operation times have SCBZ property, production time has exponential distribution and the man power system, operation time, sales and recruitment times have general distributions. Joint transforms of the variables, their means and the covariance of operation time and recruitment time with numerical results are presented.
\end{abstract}

AMS Subject Classification: $91 \mathrm{~B} 70$

Key Words: departure and recruitments, failure and repairs, production and sale times, joint transforms

\section{Introduction}

Manpower Planning models have been studied by Grinold and Marshall [2]. For statistical approach one may refer to Bartholomew [1]. Lesson [6] has given methods to compute shortages (Resignations, Dismissals, Deaths). Markovian

Received: August 27, 2015

Published: February 11, 2016

$\S$ Correspondence author (c) 2016 Academic Publications, Ltd.

url: www.acadpubl.eu 
models are designed for shortage and promotion in MPS by Vassiliou [11]. Subramanian. [10] has made an attempt to provide optimal policy for recruitment, training, promotion and shortages in manpower planning models with special provisions such as time bound promotions, cost of training and voluntary retirement schemes. For other manpower models one may refer Setlhare [9]. For three characteristics system in manpower models one may refer to Mohan and Ramanarayanan [8]. Esary et al. [3] have discussed cumulative damage processes. Stochastic analysis of manpower levels affecting business with varying recruitment rate are presented by Hari Kumar, Sekar and Ramanarayanan [4]. Manpower System with Erlang departure and one by one recruitment is discussed by Hari Kumar [5]. For the study of Semi Markov Models in Manpower planning one may refer Meclean [7].

So far various manpower models have been discussed by many researchers but man power machine system with failure, repair, product production, sales and recruitment have not been together analyzed at any depth. In real life like situation man power is required to run machines which are producing products. Due to employees' departure and due to machine failure the production system may fail calling for man power recruitment and machine repair. This makes studies on models with production, sales, recruitments and repairs are crucial for any organization and such a study is presented here.

In this paper, a machine which has a varying failure rate property known as switching the clock back to zero (SCBZ) property is considered. The man power system attending the machine is subject failure due to departure of employees. When both are in failed state repairs, recruitments, and sales begin. In this Model, man power vacancies are filled up one by one. The joint Laplace transforms of the operation time of the system, recruitment time, repair time and sale time, their means, covariance of operation and recruitment times are presented with numerical results.

\subsection{Assumptions}

1. Inter departure times of employees are independent and identically distributed ( i.i.d) random variables with $\mathrm{Cdf} F(\mathrm{x})$ and $\operatorname{pdf} \mathrm{f}(\mathrm{x})$. The manpower collapses with probability $\mathrm{p}$ when an employee leaves and with probability q manpower system survives and continues operation where $\mathrm{p}$ $+\mathrm{q}=1$.

2. The machine attended by manpower has SCBZ failure property. It has two phases. In phase I it has exponential failure time distribution with parameter 'a'. If it does not fail in an exponential time with parameter 
'c' it moves to phase 2 and the parameter 'a' changes to 'b'. It has exponential failure time distribution with parameter $b$.

3. The man power- machine system fails when both are in failed state. When either manpower or machine alone is in failed state, hiring is done to replace the failed unit till the other one also fails and both are in failed state.

4. When the system fails, the vacancies caused by the departure of employees are filled up one by one with recruitment time $\mathrm{V}$ whose $\mathrm{Cdf}$ is $\mathrm{V}(\mathrm{y})$ and pdf is $\mathrm{v}(\mathrm{y})$.

5. When the machine fails (with parameter 'a') before the change of parameter, its repair time Cdf is $R_{1}(z)$ and pdf is $r_{1}(z)$. When it fails after the change of parameter (with parameter b), its repair time $\mathrm{Cdf}$ is $\mathrm{R}_{2}(\mathrm{z})$ and pdf is $r_{2}(z)$.

6. When the man power-machine system is in operation, products are produced for sales one by one. The inter production times of products have exponential distribution with parameter $\mu$.

7. The sale times of products are i.i.d random variables $\mathrm{G}$ with $\mathrm{Cdf} \mathrm{G}(\mathrm{w})$ and pdf $\mathrm{g}(\mathrm{w})$.

8. When the man-power machine system fails, the sale time, recruitment time and repair begin.

\subsection{Analysis}

To study the above model the probability density function and the distribution of SCBZ machine life time is required. Since the parameter ' $a$ ' changes to 'b' in an exponential time with parameter ' $c$ ' if the machine does not fail in phase 1 , the life time pdf of machine satisfies the following equation.

$$
h(x)=a e^{-a x} e^{-c x}+\int_{0}^{x} c e^{-c u} e^{-a u} b e^{-b(x-u)} d u .
$$

The first term of (1) of R.H.S is the pdf part that the machine fails in phase 1 before the change in parameter. The second term is the pdf part that the machine moves to phase 2 at time $u$, no failure occurs in phase 1 and the machine fails at $\mathrm{x}$ in phase 2 . 
On simplification, the pdf of failure time of the machine is

$$
h(x)=a e^{-x(a+c)}+\frac{c b}{c+a-b}\left(e^{-b x}-e^{-(c+a) x}\right) .
$$

The first term of (2) is phase 1 failure density and the second term of (2) is phase 2 failure density. The survival probability function $\mathrm{s}(\mathrm{x})$ of the machine that it does not fail in phase 1 or in phase 2 satisfies the following equation

$$
s(x)=e^{-x(a+c)}+\int_{0}^{x} e^{-a u} c e^{-c u} e^{-b(x-u)} d u .
$$

The first term of right side of equation (3) is the probability that the machine survives in phase 1 and second term is the probability that the machine survives in phase 2. On simplification it may be obtained with two probabilities of phase 1 and phase 2 as follows

$$
s(x)=e^{-x(a+c)}+\frac{c}{c+a-b}\left(e^{-b x}-e^{-(a+c) x}\right) .
$$

Simplifying equations (2) and (4), it can be seen as

$$
h(x)=\alpha(c+a) e^{-x(a+c)}+\beta b e^{-b x},
$$

and

$$
s(x)=\alpha e^{-x(a+c)}+\beta e^{-b x},
$$

Here

$$
\alpha=\frac{a-b}{c+a-b}, \quad \beta=\frac{c}{c+a-b} \quad \text { and } \quad \alpha+\beta=1 .
$$

The equations (2) and (4) present the failure or survival function in phase 1 and phase 2 explicitly for repair and maintenance study.

The joint probability density function of four variables, namely $(X, \hat{V}, \hat{R}, \hat{S})$ is required for the study of the models.

Here:

(i) $X$ is the manpower-machine system operation time;

(ii) $\hat{V}$ is the total recruitment time of employees

(iii) $\hat{R}$ is the repair time or the maintenance time of the machine and

(iv) $\hat{S}$ is the total sales time of the products. 
When $k_{1}$ employees have left and $k_{2}$ products produced then $\hat{V}=V_{1}+V_{2}+$ $\ldots . V_{k_{1}}$ and $\hat{S}=G_{1}+G_{2}+\ldots . G_{k_{2}}$.

The repair time $\hat{R}$ is either $R_{1}$ or $R_{2}$ according as the machine fails in phase 1 or fails in phase 2 . We also note that $\mathrm{X}$ is the maximum of the life times of both manpower and machine systems.

We find the pdf $f(x, y, z, w)$ of $(X, \hat{V}, \hat{R}, \hat{S})$ as follows

$$
\begin{array}{r}
f(x, y, z, w)=\left\{\left[a e^{-x(a+c)} r_{1}(z)+\frac{c b}{c+a-b}\left(e^{-b x}-e^{-(c+a) x}\right) r_{2}(z)\right]\right. \\
\times\left[\sum_{n=1}^{\infty} F_{n}(x) p q^{n-1} v_{n}(y)\right] \\
+\left[r_{1}(z) \int_{0}^{x} a e^{-u(a+c)} d u+r_{2}(z) \frac{c b}{c+a-b} \int_{0}^{x}\left(e^{-b u}-e^{-u(a+c)}\right) d u\right. \\
\left.\times\left[\sum_{n=1}^{\infty} f_{n}(x) q^{n-1} p v_{n}(y)\right]\right\}\left[\sum_{k=0}^{\infty} e^{-\mu x} \frac{(\mu x)^{k}}{k !} g_{k}(w)\right] .
\end{array}
$$

Here $v_{n}(y), f_{n}(x)$ and $g_{k}(w)$ are $n$ fold convolution of $v(y), f(x)$ and $k$ fold convolution of $\mathrm{g}(\mathrm{w})$ with itself respectively. $\mathrm{F}_{\mathrm{n}}(\mathrm{x})$ is the $\mathrm{n}$-fold Stiltjes convolution of $\operatorname{Cdf} \mathrm{F}(\mathrm{x})$ with itself.

To write down the equation (8) the two cases namely (i) the machine fails when the manpower system is in failed state and (ii) the manpower system fails when the machine is in failed state are considered. They are given as two terms inside the flower bracket. The first term has two square brackets. The first square bracket is presented using the terms of equation (2) multiplied by phase 1 and phase 2 failure's repair densities when the machine fails. The second square bracket is the case of presenting the manpower system already in failed state on the $\mathrm{n}$-th departure and the recruitments for them are done one by one. The second term has two square brackets of which the first one is presented when the machine is already in failed state, the manpower collapses on the n-th departure of an employee. The phase 1 or phase 2 failures, repair densities and the density for $\mathrm{n}$ recruitments are presented there to indicate they are done. The last square bracket indicates the k products produced are sold one by one. $\hat{R}, \hat{S})$

We now present the quadruple Laplace transform of the joint pdf of $(X, \hat{V}$,

$$
f^{*}(\xi, \eta, \varepsilon, \delta)=\int_{0}^{\infty} \int_{0}^{\infty} \int_{0}^{\infty} \int_{0}^{\infty} e^{-\xi x-\eta y-\varepsilon z-\delta w} f(x, y, z, w) d x d y d z d w
$$


Because of the structure of equation (8) the equation (9) becomes a single integral as follows.

$$
\begin{gathered}
f^{*}(\xi, \eta, \varepsilon, \delta)=\int_{0}^{\infty} e^{-\xi x} e^{-\mu x\left(1-g^{*}(\delta)\right)}\left\{\left[a e^{-x(a+c)} r_{1}^{*}(\varepsilon)\right.\right. \\
\left.\quad+\left(\frac{c b}{c+a-b}\right)\left(e^{-b x}-e^{-(c+a) x}\right) r_{2}^{*}(\varepsilon)\right] \\
\times\left[\sum_{n=1}^{\infty} F_{n}(x) p q^{n-1} v^{*^{n}}(\eta)\right]+\left[r_{1}^{*}(\varepsilon) \frac{a}{a+c}\left(1-e^{-x(a+c)}\right)+r_{2}^{*}(\varepsilon)\left(\frac{c b}{c+a-b}\right)\right. \\
\left.\left.\left(\frac{1-e^{-b x}}{b}-\frac{1-e^{-(c+a) x}}{c+a}\right)\right]\left[\sum_{n=1}^{\infty} f_{n}(x) q^{n-1} p v^{*^{*}}(\eta)\right]\right\} d x .
\end{gathered}
$$

This simplifies as follows.

$$
\begin{array}{r}
f^{*}(\xi, \eta, \varepsilon, \delta)=\left(\frac{1}{\chi_{1}}\left[a r_{1}^{*}(\varepsilon)-\frac{c b}{c+a-b} r_{2}^{*}(\varepsilon)\right]-\frac{a}{a+c} r_{1}^{*}(\varepsilon)+\frac{c b}{c+a-b} \frac{1}{c+a} r_{2}^{*}(\varepsilon)\right) \\
\left.\left(\frac{p f^{*}\left(\chi_{1}\right) v^{*}(\eta)}{\left(1-q v^{*}(\eta) f^{*}\left(\chi_{1}\right)\right)}\right)+\left(\frac{1}{\chi_{2}}\left(\frac{c b}{c+a-b}\right) r_{2}^{*}(\varepsilon)-\left(\frac{c}{c+a-b}\right) r_{3}^{*}(\varepsilon)\right)\right) \\
\left(\frac{p f^{*}\left(\chi_{2}\right) v^{*}(\eta)}{\left(1-q v^{*}(\eta) f^{*}\left(\chi_{2}\right)\right)}\right)+\left(\frac{a}{a+c} r_{1}^{*}(\varepsilon)+r_{2}^{*}(\varepsilon) \frac{c}{c+a}\right)\left(\frac{p f^{*}\left(\chi_{3}\right) v^{*}(\eta)}{\left(1-q v^{*}(\eta) f^{*}\left(\chi_{3}\right)\right)}\right) .
\end{array}
$$

Here

$$
\begin{aligned}
& \chi_{1}=\xi+\mu\left(1-g^{*}(\delta)\right)+a+c \\
& \chi_{2}=\xi+\mu\left(1-g^{*}(\delta)\right)+b \\
& \chi_{3}=\xi+\mu\left(1-g^{*}(\delta)\right) .
\end{aligned}
$$

Now the Laplace transform of the pdf of $X$ is

$$
\begin{gathered}
f^{*}(\xi, 0,0,0)=\frac{p f^{*}(\xi)}{1-q f^{*}(\xi)}-\frac{\alpha \xi}{(\xi+a+c)} \frac{p f^{*}(\xi+a+c)}{\left(1-q f^{*}(\xi+a+c)\right)} \\
-\frac{\beta \xi}{(\xi+b)} \frac{p f^{*}(\xi+b)}{\left(1-q f^{*}(\xi+b)\right)} \\
E(X)=-\frac{\partial}{\partial \xi} f^{*}(\xi, 0,0,0) \quad \text { at } \quad \xi=0 .
\end{gathered}
$$

This gives

$$
E(X)=\frac{E(F)}{p}+\frac{\alpha}{(a+c)} \frac{\left.p f^{*}(a+c)\right)}{\left(1-q f^{*}(a+c)\right)}+\frac{\beta p}{b} \frac{f^{*}(b)}{\left(1-q f^{*}(b)\right)} .
$$


The Laplace transform of pdf of $\hat{V}$ is

$$
\begin{gathered}
f^{*}(0, \eta, 0,0)=\frac{p v^{*}(\eta)}{1-q v^{*}(\eta)}, \\
E(\hat{V})=\frac{\partial}{\partial \eta} f^{*}(0, \eta, 0,0) \mid \eta=0 .
\end{gathered}
$$

Therefore

$$
E(\hat{V})=\frac{E(V)}{p} .
$$

The Laplace transform of repair time of the machine is

$$
\begin{gathered}
f^{*}(0,0, \varepsilon, 0)=\left(\frac{a}{a+c}\right) v_{1}^{*}(\varepsilon)+\left(\frac{c}{c+a}\right) v_{2}^{*}(\varepsilon), \\
E(\hat{R})=-\left.\frac{\partial}{\partial \varepsilon} f^{*}(0,0, \varepsilon, 0)\right|_{\varepsilon=0} .
\end{gathered}
$$

Therefore

$$
E(\hat{R})=\frac{a}{a+c} E\left(R_{1}\right)+\frac{c}{a+c} E\left(R_{2}\right) .
$$

The Laplace transform of the sales time is

$$
\begin{aligned}
& f^{*}(0,0,0, \delta)=\frac{-\alpha \mu\left(1-g^{*}(\delta)\right)}{\left(\mu\left(1-g^{*}(\delta)\right)+a+c\right)}-\frac{\beta \mu\left(1-g^{*}(\delta)\right)}{\left(\mu\left(1-g^{*}(\delta)\right)+b\right)} \\
& +\frac{p f^{*}\left(\mu\left(1-g^{*}(\delta)\right)\right.}{\left(1-q f^{*}\left(\mu\left(1-g^{*}(\delta)\right)\right)\right)} \text {. }
\end{aligned}
$$

The expected sales time is

$$
E(\hat{S})=E(G) \mu\left[\frac{\alpha}{a+c}+\frac{\beta}{b}+\frac{E(F)}{p}\right] .
$$

The joint Laplace transform of Operation time and recruitment time $(X, \hat{V})$ is given by

$$
\begin{array}{r}
f^{*}(\xi, \eta, 0,0)=\frac{p f^{*}(\xi) v^{*}(\eta)}{\left(1-q f^{*}(\xi) v^{*}(\eta)\right)}-\frac{\alpha \xi}{(\xi+a+c)} \frac{p v^{*}(\eta) f^{*}(\xi+a+c)}{\left(1-q v^{*}(\eta) f^{*}(\xi+a+c)\right)} \\
-\frac{\beta \xi}{(\xi+b)} \frac{p v^{*}(\eta) f^{*}(\xi+b)}{\left(1-q v^{*}(\eta) f^{*}(\xi+b)\right)}
\end{array}
$$

The product moment $E(X \hat{V})$ is given by 


$$
\begin{aligned}
& E(X \hat{V})=\frac{\partial^{2}}{\partial \xi \partial \eta} f^{*}(\xi, \eta, 0,0) \mid \xi=\eta=0 E(X \hat{V}) \\
= & E(V)\left[\frac{(1+q)}{p^{2}} E(F)+\frac{\alpha p}{(a+c)} \frac{f^{*}(a+c)}{\left(1-q f^{*}(a+c)\right)^{2}}+\frac{\beta p}{b} \frac{f^{*}(b)}{\left(1-q f^{*}(b)\right)^{2}}\right] .
\end{aligned}
$$

Using the formula $\operatorname{Cov}(X \hat{V})=E(X \hat{V})-E(X) E(\hat{V})$ we can re-write the covariance using equations (22), (16) and (14).

\section{Numerical Examples}

The usefulness of the results obtained is presented by numerical examples.

Let $a=15, b=5, c=10, p=0.4, q=0.6, E(F)=10, E\left(R_{1}\right)=15$, $E\left(R_{2}\right)=10, E(G)=12, E(V)=10, \mu=5,10,15,20,25$ and $\delta=2,4,6,8,10$.

\begin{tabular}{|c|c|c|c|c|c|c|}
\hline$\mu / \delta$ & 2 & 4 & 6 & 8 & 10 & 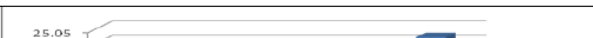 \\
\hline 5 & 25.01441 & 25.02545 & 25.03418 & \begin{tabular}{|l|}
25.04129 \\
\end{tabular} & 25.0472 & $\begin{array}{l}25.05 \\
25.04 \\
25.04\end{array}$ \\
\hline 10 & 25.01441 & 25.02545 & 25.03418 & \begin{tabular}{|l|}
25.04129 \\
\end{tabular} & 25.0472 & $\begin{aligned} 25.035 \\
25.03 \\
25.03\end{aligned}$ \\
\hline 15 & 25.01441 & 25.02545 & 25.03418 & \begin{tabular}{|l|l|}
25.04129 \\
\end{tabular} & 25.0472 & - Series1 \\
\hline 20 & 25.01441 & 25.02545 & 25.03418 & \begin{tabular}{|l|}
25.04129 \\
\end{tabular} & 25.0472 & $\begin{array}{l}25015 \\
25.01 \\
25.01\end{array}$ \\
\hline 25 & 25.01441 & 25.02545 & 25.03418 & \begin{tabular}{|l|l|}
25.04129 \\
\end{tabular} & 25.0472 & \\
\hline
\end{tabular}

Here " $\mathrm{f}$ " is an exponential density function with parameter " $\delta$ ".

Table 1: Table and graph of $\mathrm{E}(\mathrm{X})$

When " $\delta$ " increases the expected operation time $\mathrm{E}(\mathrm{X})$ increases.

\begin{tabular}{|c|r|r|r|r|r|r|r|}
\hline$\mu / \delta$ & 2 & 4 & 6 & 8 & 10 & \\
\hline 5 & 13 & 13 & 13 & 13 & 13 \\
\hline 10 & 13 & 13 & 13 & 13 & 13 \\
\hline 15 & 13 & 13 & 13 & 13 & 13 \\
\hline 20 & 13 & 13 & 13 & 13 & 13 \\
\hline 25 & 13 & 13 & 13 & 13 & 13 \\
\hline
\end{tabular}

Table 2: The table and graph for $E(\hat{V})$

Irrespective of the value of $\mu$ and $\delta, E(\hat{V})$ remains constant.

When $\mu$ increases the expected repair time $E(\hat{R})$ increases. 


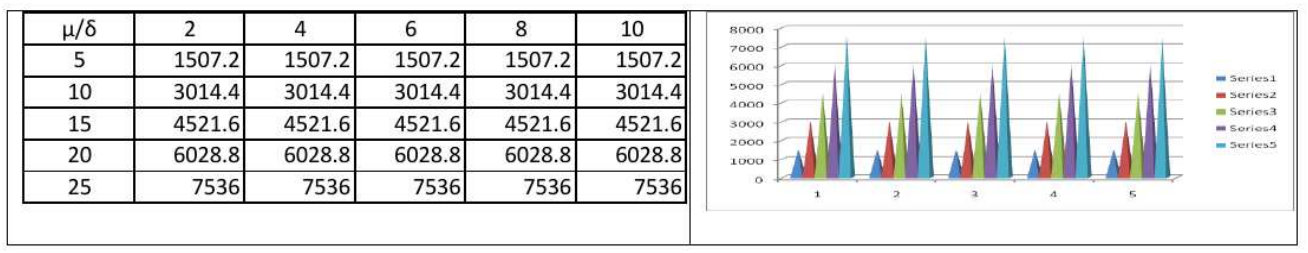

Table 3: The table and graph for $E(\hat{R})$

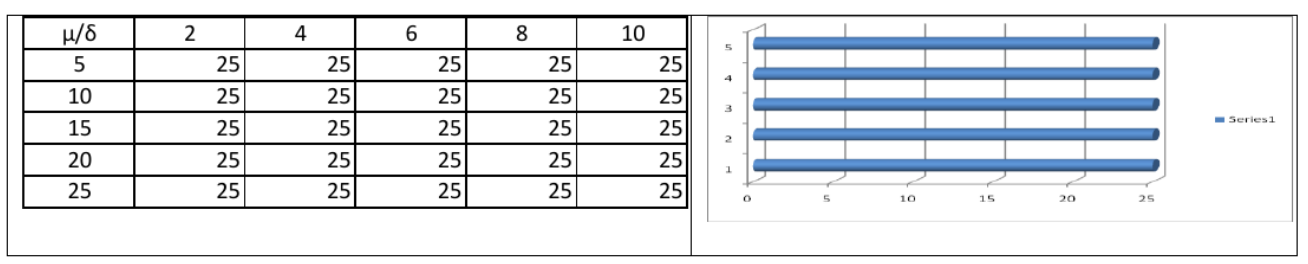

Table 4: The table and graph for $E(\hat{S})$

\begin{tabular}{|c|c|c|c|c|c|c|}
\hline$\mu / \delta$ & 2 & 4 & 6 & 8 & 10 & $\begin{array}{l}1000.9 \\
1000.8\end{array}$ \\
\hline 5 & 1000.173 & 1000.344 & 1000.502 & 1000.645 & 1000.774 & $\begin{array}{l}1000.7 \\
1000.6\end{array}$ \\
\hline 10 & 1000.173 & 1000.344 & 1000.502 & 1000.645 & 1000.774 & 1000.5 \\
\hline 15 & 1000.173 & 1000.344 & 1000.502 & 1000.645 & 1000.774 & 1000.3 \\
\hline 20 & 1000.173 & 1000.344 & 1000.502 & 1000.645 & 1000.774 & $\begin{array}{l}1000.1 \\
1000.1\end{array}$ \\
\hline 25 & 1000.173 & 1000.344 & 1000.502 & 1000.645 & 1000.774 & $\begin{array}{l}1000 \\
999.9\end{array}$ \\
\hline
\end{tabular}

Table 5: The table and graph for $E(X \hat{V})$

Irrespective of the value of $\mu$ and $\delta, E(\hat{S})$ remains constant.

When $\delta$ increases $E(X \hat{V})$ increases.

When $\delta$ increases $\operatorname{Cov}(X \hat{V})$ decreases

\section{References}

[1] D.J. Barthlomew, Statistical Technique for Manpower Planning, John Wiley, Chichester (1979). 


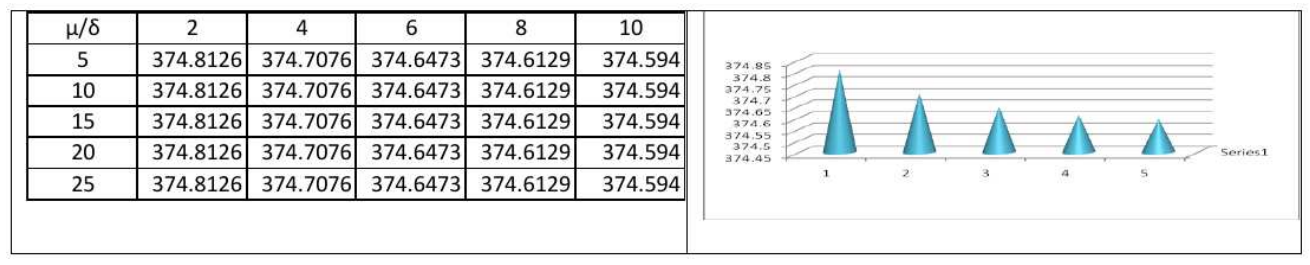

Table 6: The table and graph for $\operatorname{Cov}(X \hat{V})$

[2] J.D. Esary, A.W. Marshall, F. Proschan, Shock models and wear processes, Ann. Probability, 1, No. 4 (1973), 627-649.

[3] R.C. Grinold, K.T. Marshall, Manpower Planning Models, North Holl, Newyork (1977).

[4] K. Hari Kumar, P. Sekar, R. Ramanarayanan, Stochastic analysis of manpower levels affecting business with varying recruitment rate, International Journal of Applied Mathematics, 8, No. 29 (2014), 1421-1428.

[5] K. Hari Kumar, Manpower system with erlang departure and one by one recruitment, International Journal of Mathematical Archive, 5, No. 7 (2014), 89-92.

[6] G.W. Lesson, Wastage and promotion in desired manpower structures, J. Opl. Res. Soc., 33 (1982), 433-442.

[7] McClean, Semi-Markovian models in continuous time, J. Appl. Prob., 16 (1980), 416-422.

[8] C. Mohan, R. Ramanarayanan, An analysis of manpower, money and business with random environments, International Journal of Applied Mathematics, 23, No. 5 (2010), 927-940.

[9] Setlhare, Modeling of an intermittenly busy manpower system, In: Proceedings at the Conference held in Sept., 2006 at Gabarone, Botswana (2007).

[10] V. Subramanian, Optimum promotion rate in manpower models, International Journal of Management and System, 12, No. 2 (1996), 179-184.

[11] P.C.G. Vassiliou, A higher order Markovian model for prediction of wastage in manpower system, Operat. Res. Quart., 27 (1976). 